\title{
Paraneoplastic Dermatomyositis Syndrome Presenting as Dysphagia
}

\author{
Emmanuel Ofori $^{\mathrm{a}}$, Daryl Ramai ${ }^{\mathrm{b}, \mathrm{c}}$, Mel Ona ${ }^{\mathrm{a}}$, Madhavi Reddy $^{\mathrm{a}}$
}

\begin{abstract}
Dermatomyositis (DM) is a rare autoimmune condition which predominantly affects females in the fifth and sixth decades of life. DM presents acutely or progressively as painless proximal skeletal muscle weakness and can be associated with a heliotropic rash around the eyes, and Gottron's papules on extensor surfaces of the hands. While the pathophysiology of DM is still unclear, abnormal T- and B-cell immune activity has been reported. DM has been associated with malignancy and has been shown to appear before, concurrently with, or after diagnosis of cancer. We report a 72-year-old female with renal clear cell carcinoma, breast cancer, and papillary serous carcinoma of the uterus, who presented with progressive weakness, 30 pound weight loss, and dysphagia over the past 3 months. She also reported difficulty in ambulating, facial rash, and inability to extend her fingers. Left bicep muscle biopsy and staining with nicotinamide adenine dinucleotide tetrazolium reductase (NADH-TR) identified frequent myofibers with targetoidlike changes consistent with DM. Despite immunosuppression with intravenous corticosteroids, she did not respond to the treatment and a percutaneous gastrostomy tube was placed for enteral feeding.
\end{abstract}

Keywords: Dermatomyositis; Dysphagia; Paraneoplastic syndrome; Breast cancer; Uterine carcinoma; Renal clear cell carcinoma

\section{Introduction}

Dermatomyositis (DM) is a rare idiopathic inflammatory muscle and skin disorder that afflicts two in every 100,000 individuals of the population annually [1]. DM occurs more often in women with peak incidence in the fifth and sixth decades of life $[2,3]$. DM presents acutely or progressively as painless proximal skeletal muscle weakness and can be associated with

Manuscript submitted April 26, 2017, accepted May 23, 2017

aDepartment of Gastroenterology and Hepatology, The Brooklyn Hospital Center, Academic Affiliate of The Icahn School of Medicine at Mount Sinai, Clinical Affiliate of The Mount Sinai Hospital, 121 Dekalb Avenue, Brooklyn, NY 11201, USA

bepartment of Anatomical Sciences, St. George's University School of Medicine, True Blue, Grenada, WI

${ }^{\mathrm{c} C}$ Corresponding Author: Daryl Ramai, Department of Anatomical Sciences, St. George's University School of Medicine, True Blue, Grenada, WI.

Email: dramai@sgu.edu a characteristic heliotropic rash around the eyes and Gottron's papules on the extensor surface of the hands [4]. Individuals may also present with symptoms of dysphagia in addition to having pulmonary and cardiac involvement [5]. The association between DM and malignancy has been widely established over the years and may appear before, concurrent with, or after diagnosis of cancer $[6,7]$. Here in, we describe a case of a 72-year-old woman with a history of malignancies who presented with dysphagia which illustrates a paraneoplastic course (evolving after cancer was diagnosed). In addition, we review current knowledge on the history, pathophysiology, and treatment of malignancy associated DM.

\section{Case Report}

A 72-year-old female with medical history of renal clear cell carcinoma, breast cancer (status post lumpectomy and radiotherapy), and papillary serous carcinoma of uterus (pT2N0Mx - status post total abdominal hysterectomy and bilateral salpingo-oophrectomy, and omentectomy status post chemotherapy completed 5 months prior) presented with a complaint of dysphagia. Our patient reported about 4 - 6 weeks duration of poor appetite and progressive weakness which rapidly deteriorated 4 days prior to admission. She experienced difficulty in swallowing both liquids and solid foods with an associated thirty pound unintentional weight loss over a 3-month duration. Physical examination revealed a chronically ill appearing woman with areas of hyperpigmentation on the head (Fig. 1),

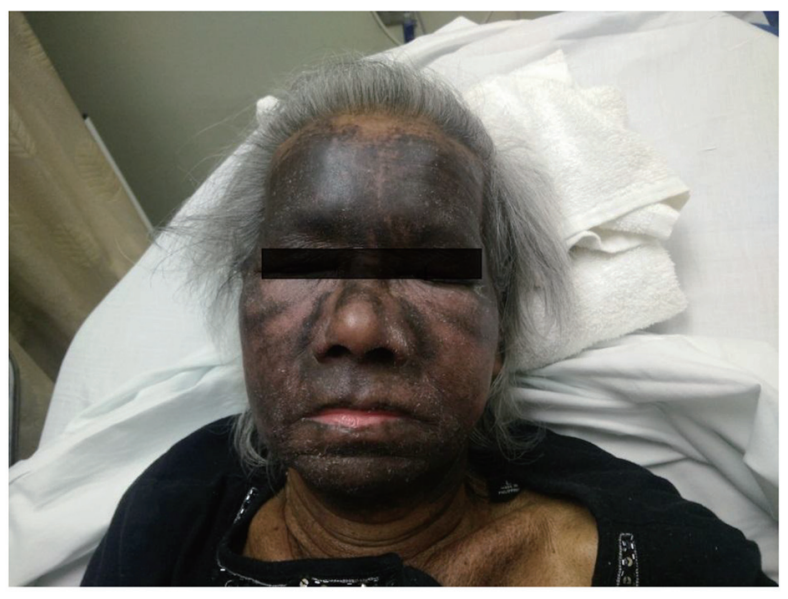

Figure 1. Facial hyperpigmentation. 


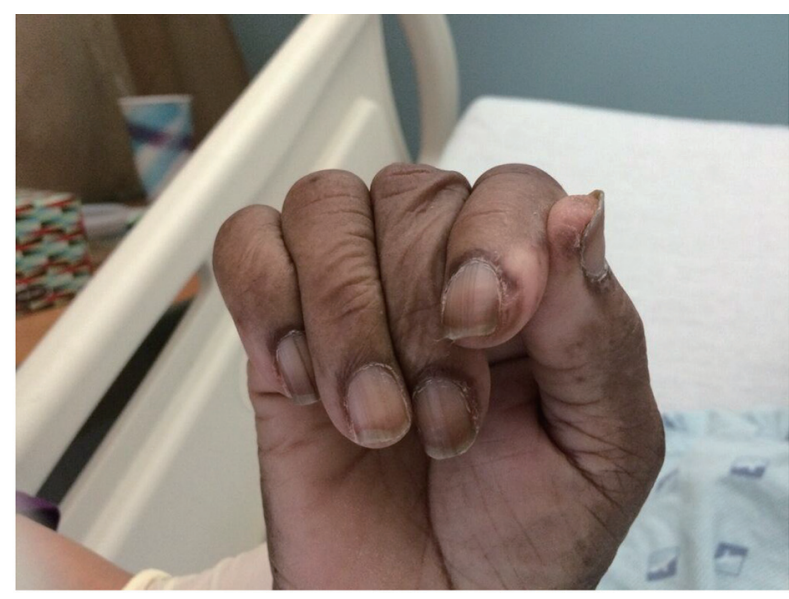

Figure 2. Periungual hyperemia.

arms and legs without a periorbital heliotropic rash. The metacarpophalangeal joints and proximal interphalangeal joints were flexed bilaterally, without scaling or Gottron's papules, and distal phalanges with periungual hyperemia (Fig. 2). Our patient had symmetric proximal muscle weakness, $4 / 5$ power in the upper extremities, and $2 / 5$ power in the lower extremities.

Laboratory findings were significant for an elevated serum creatinine phosphokinase (CPK) level of 3,222 U/L, creatine kinase isoform MB $369.7 \mathrm{ng} / \mathrm{mL}$, aldolase $31.7 \mathrm{U} / \mathrm{L}$, erythrocyte sedimentation rate $40 \mathrm{~mm} / \mathrm{h}$, C-reactive protein 8.41 $\mathrm{mg} / \mathrm{L}$, lactate dehydrogenase (LDH) $804 \mathrm{U} / \mathrm{L}$, and antinuclear antibody positive $>1: 640$. Barium esophagram revealed significant difficulty in initiating swallow with laryngeal penetration and aspiration of contrast material.

Esophagogastroduodenoscopy showed pooling of saliva in the piriform fossa with a normal appearing esophagus (Fig. 3). Random esophageal biopsies returned with the final pathology of squamous esophageal mucosa with mild chronic non-specific esophagitis and hyperkeratosis. Subsequently, a left biceps muscle biopsy was performed and revealed inflam-

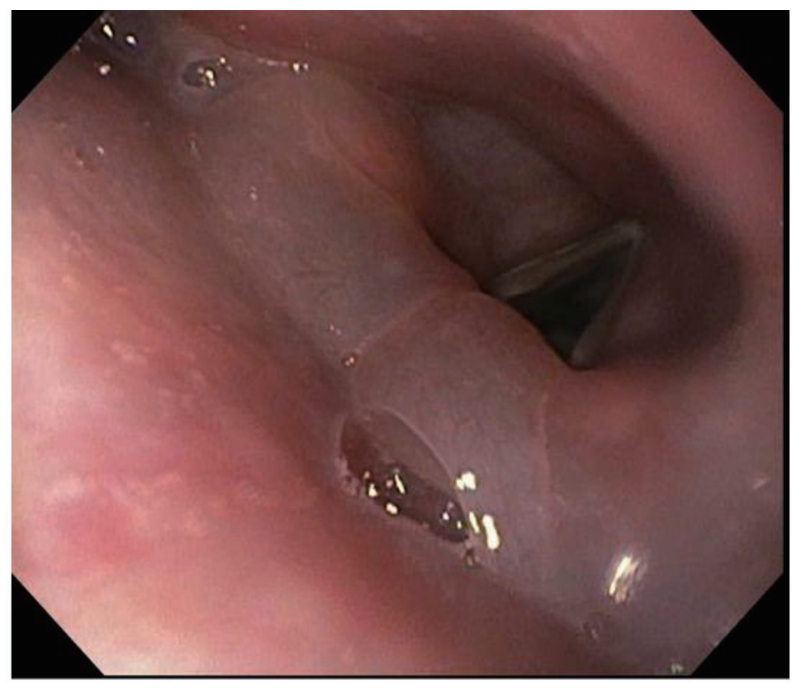

Figure 3. Pooling of saliva in the piriform fossa.

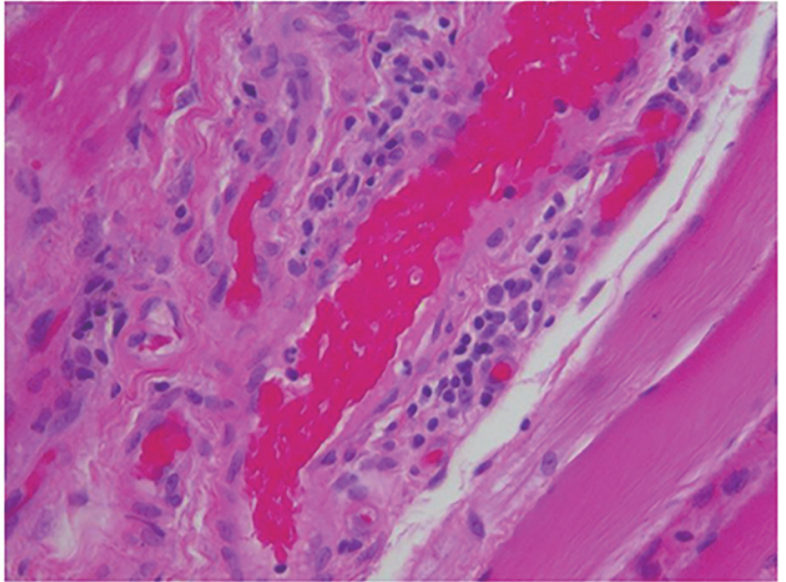

Figure 4. Inflammatory myopathic process with significant necrotizing component $(\times 40)$.

matory myositis with severe necrotizing component suggestive of DM (Fig. 4). Further staining with nicotinamide adenine dinucleotide tetrazolium reductase (NADH-TR) stain identified frequent myofibers with targetoid-like changes consistent with DM (Fig. 5). Our patient was treated with intravenous corticosteroids; however, she did not respond to the treatment. Thus, a percutaneous gastrostomy tube was placed for enteral feeding.

\section{Discussion}

DM is a rare autoimmune myopathy with cutaneous manifestations. It usually presents as painless, proximal muscle weakness, with or without the pathognomonic skin involvements of heliotrope rash and Gottron's papules [4]. DM may also present with mastication difficulties, dysarthria and dysphagia [4]. Approximately $10-73 \%$ of patients with inflammatory myopathy experience dysphagia [8]. When this condition presents without any cutaneous disease, it is known as polymositis (PM) [9]. More subtly, adermatopathic DM is histopathologi-

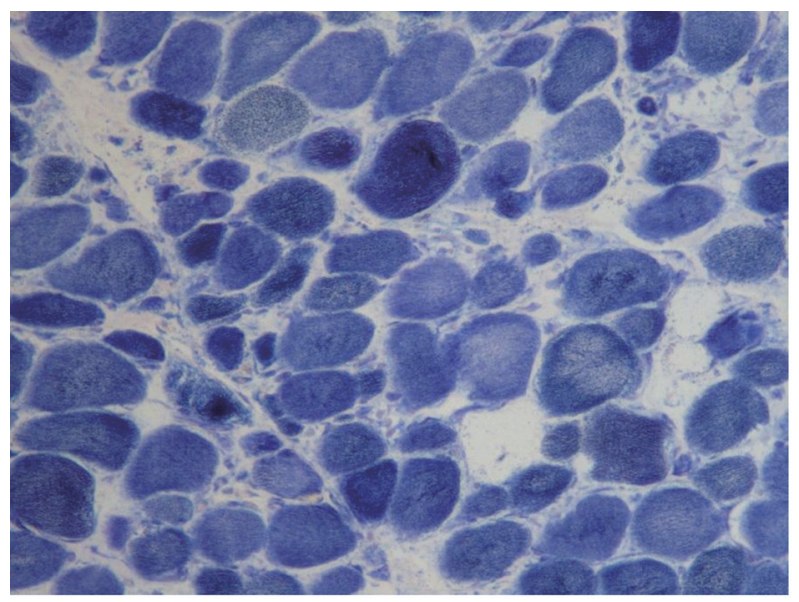

Figure 5. Myofibers with targetoid-like changes are identified with NADH-TR stain $(\times 100)$. 
cally proven DM without the classic rash, making it difficult to diagnose [4].

DM is diagnosed using a criterion of five key pathological symptoms, namely 1) progressive symmetric weakness of proximal limbs and anterior flexor muscles of the neck, 2) dermatological signs including heliotropic rash, poikiloderma, Grotton's papules, and dermatitis on elbows, knees or feet, 3) necrosis of type 1 and 2 on muscle biopsy, 4) abnormal electromyography, and 5) elevated muscle enzyme levels (CPK, aldolase, and LDH) [2]. Some cases present with alterations in nail bed vascularity and periungal hyperemia due to microhemorrhages and capillary enlargement [10].

While the pathophysiology of DM is not clearly understood, an association with malignancy has been well established. Approximately $30 \%$ of DM cases are associated with an underlying malignancy, in which it is considered a paraneoplastic syndrome [11]. Cancers associated with DM include cancers of the ovary, lung, pancreas, breast, gastrointestinal tract, non-Hodgkin's lymphoma, testicular, and nasopharyngeal carcinoma particularly in the Southeast Asian population [11-13]. The most common malignancy associated with DM is ovarian cancer, though $20 \%$ of cases have been linked to breast cancer [14]. Interestingly, DM in the setting of malignancy can present before, concurrently with, or after cancer is diagnosed. Thus early symptoms of DM should prompt clinicians to be suspicious of a malignant etiology.

The classic histological view of DM looks for immune complexes which are deposited at dermal-epidermal junctions. Elevated T and B cells are also observed accumulating in perivascular spaces [15]. However, the pathophysiology of DM and malignancy may be different. One theory suggests that as tumors express oncoproteins, it stimulates an inflammatory immune response which deposits autoantibodies where skin and muscle antigens are found. Evidently, 30\% of patients have myositis-specific antibodies such as anti-synthetases, anti-signal recognition particle antibodies, and anti-Mi-2 antibodies [16].

The treatment for DM or PM requires a long-term course of oral immunosuppression with corticosteroids. In cases with an underlying malignancy, therapy is variable and often more resistant to suppression. However, adding intravenous immunoglobulins (IVIG) has been reported to be helpful [17]. Additional third line drugs are available such as mycophenolate mofetil, cyclosporine, tacrolimus, rituximab, etanercept and cyclophosphamide [4]. If possible, surgical resection of the underlying cancer can provide dramatic improvement in both cutaneous and musculoskeletal symptoms [18]. While our patient has three different forms of cancer, it is difficult to identify which cancer was responsible for her symptoms. Evidently, our patient's condition did not respond to corticosteroids, a signal of poor prognosis, and a percutaneous gastrostomy tube was placed for enteral feeding.

\section{Conclusion}

$\mathrm{DM}$ is a rare autoimmune condition which affects skin and muscles. A small subset of cases is associated with an underlying malignancy. We report a very rare case of paraneoplas- tic DM presenting with dysphagia unresponsive to treatment. Symptoms of progressive dysphagia in patients with muscle weakness can be a hallmark of progressive DM secondary to infiltrating conditions and malignancies.

\section{Conflicts of Interest}

The authors have no conflicts of interest or financial relationships to disclose.

\section{References}

1. Jacobson DL, Gange SJ, Rose NR, Graham NM. Epidemiology and estimated population burden of selected autoimmune diseases in the United States. Clin Immunol Immunopathol. 1997;84(3):223-243.

2. Bohan A, Peter JB, Bowman RL, Pearson CM. Computer-assisted analysis of 153 patients with polymyositis and dermatomyositis. Medicine (Baltimore). 1977;56(4):255286.

3. Tymms KE, Webb J. Dermatopolymyositis and other connective tissue diseases: a review of 105 cases. J Rheumatol. $1985 ; 12(6): 1140-1148$.

4. Dimachkie MM, Barohn RJ. Idiopathic inflammatory myopathies. Semin Neurol. 2012;32(3):227-236.

5. Dagan A, Markovits D, Braun-Moscovici Y, Rozin A, Toledano K, Balbir-Gurman A. Life-threatening oropharyngeal aphagia as the major manifestation of dermatomyositis. Isr Med Assoc J. 2013;15(8):453-455.

6. Samuels N, Applbaum YH, Esayag Y. Paraneoplastic necrotizing myopathy and dermatomyositis in a patient with rectosigmoid carcinoma. Rheumatol Int. 2013;33(6):1619-1621.

7. Osako T, Ito Y, Morimatsu A, Tada K, Sakurai N, Takahashi S, Akiyama F, et al. Flare-up of dermatomyositis along with recurrence of breast cancer. Breast J. 2007;13(2):200-202.

8. Oh TH, Brumfield KA, Hoskin TL, Stolp KA, Murray JA, Bassford JR. Dysphagia in inflammatory myopathy: clinical characteristics, treatment strategies, and outcome in 62 patients. Mayo Clin Proc. 2007;82(4):441-447.

9. Callen JP, Wortmann RL. Dermatomyositis. Clin Dermatol. 2006;24(5):363-373.

10. Selva-O'Callaghan A, Fonollosa-Pla V, Trallero-Araguas E, Martinez-Gomez X, Simeon-Aznar CP, Labrador-Horrillo M, Vilardell-Tarres M. Nailfold capillary microscopy in adults with inflammatory myopathy. Semin Arthritis Rheum. 2010;39(5):398-404.

11. Maoz CR, Langevitz P, Livneh A, Blumstein Z, Sadeh M, Bank I, Gur H, et al. High incidence of malignancies in patients with dermatomyositis and polymyositis: an 11year analysis. Semin Arthritis Rheum. 1998;27(5):319324.

12. Hill CL, Zhang Y, Sigurgeirsson B, Pukkala E, Mellemkjaer L, Airio A, Evans SR, et al. Frequency of specific cancer types in dermatomyositis and polymyositis: a population-based study. Lancet. 2001;357(9250):96-100. 
13. Hu WJ, Chen DL, Min HQ. Study of 45 cases of nasopharyngeal carcinoma with dermatomyositis. Am J Clin Oncol. 1996;19(1):35-38.

14. Callen JP, Hyla JF, Bole GG, Jr., Kay DR. The relationship of dermatomyositis and polymyositis to internal malignancy. Arch Dermatol. 1980;116(3):295-298.

15. Kissel JT, Mendell JR, Rammohan KW. Microvascular deposition of complement membrane attack complex in dermatomyositis. N Engl J Med. 1986;314(6):329-334.

16. Cruellas MG, Viana Vdos S, Levy-Neto M, Souza FH, Shinjo SK. Myositis-specific and myositis-associated autoantibody profiles and their clinical associations in a large series of patients with polymyositis and dermatomyositis. Clinics (Sao Paulo). 2013;68(7):909-914.

17. Joshi D, Mahmood R, Williams P, Kitchen P. Dysphagia secondary to dermatomyositis treated successfully with intravenous immunoglobulin: a case report. Int Arch Med. 2008;1(1):12.

18. Luu X, Leonard S, Joseph KA. Dermatomyositis presenting as a paraneoplastic syndrome with resolution of symptoms following surgical management of underlying breast malignancy. J Surg Case Rep. 2015;2015(7):rjv075. 